\title{
THE WAHL MAP OF ONE-NODAL CURVES ON K3 SURFACES
}

\author{
EDOARDO SERNESI
}

This paper is dedicated to L. Ein on the occasion of his 60-th birthday.

\begin{abstract}
We consider a general primitively polarized K3 surface $(S, H)$ of genus $g+1$ and a 1-nodal curve $\widetilde{C} \in|H|$. We prove that the normalization $C$ of $\widetilde{C}$ has surjective Wahl map provided $g=40,42$ or $\geq 44$.
\end{abstract}

\section{INTRODUCTION}

In this Note we consider 1-nodal curves lying on a K3 surface and we study the gaussian map, or Wahl map, on their normalization. If we consider a primitive linear system $|H|$ on a K3 surface $S$, then it is well known that every nonsingular $\widetilde{C} \in|H|$ has a non-surjective Wahl map

$$
\Phi_{K}: \bigwedge^{2} H^{0}\left(\widetilde{C}, \omega_{\widetilde{C}}\right) \longrightarrow H^{0}\left(\widetilde{C}, \omega_{\widetilde{C}}^{3}\right)
$$

(see $₫ 1$ for the definition) and that, if moreover $\widetilde{C} \in|H|$ is general then it is BrillNoether-Petri general W1, BM, L. It is of some interest to decide whether the same properties hold for the normalization $C$ of a 1-nodal $\widetilde{C} \in|H|$, and more generally for the normalization of a singular $\widetilde{C}$ in $|H|$. The Brill-Noether theory of singular curves on a $\mathrm{K} 3$ surface has received quite a lot of attention in recent times, see e.g. Go, FKP, BFT, CK, Ke. On the other hand to our knowledge very little is known on their Wahl map. In $\mathrm{Hal2}, \mathrm{Ke}$ the authors consider a modified version of the Wahl map, which does not seem to have a direct and simple relation with the ordinary Wahl map $\Phi_{K}$; in particular their results point towards the nonsurjectivity of such modified map. A different point of view is taken in $\underline{\mathrm{BF}}$, where the authors give necessary conditions for a singular curve to be hyperplane section of a smooth surface, again in terms of non-surjectivity of certain maps. On the other hand in FKPS it is proved that the normalization $C$ (of genus 10) of a general 1-nodal curve $\widetilde{C} \in|H|$ on a general polarized $(S, H)$ of genus 11 has general moduli; then the main result of $\mathrm{CHM}$ implies that $\Phi_{K}$ is surjective for such a curve. This surjectivity result is extended in the present paper in the following form:

Theorem 1. Let $(S, H)$ be a general primitively polarized $K 3$ surface of genus $g+1$. Assume that $g=40,42$ or $\geq 44$. Let $\widetilde{C} \in|H|$ be a 1-nodal curve and $C$ its

2010 Mathematics Subject Classification. Primary 14J28, 14H10; Secondary 14H51.

Key words and phrases. K3 surface, Wahl map.

We thank M. Halic and M. Kemeny for helpful e-mail correspondence, A. L. Knutsen and A. Bruno for very useful comments on a preliminary version of this work, and the referee for his careful report. The author is member of GNSAGA-INDAM. 
normalization. Then the Wahl map

$$
\Phi_{K}: \bigwedge^{2} H^{0}\left(C, \omega_{C}\right) \longrightarrow H^{0}\left(C, \omega_{C}^{3}\right)
$$

is surjective.

This of course gives another proof of the main result of [CHM] for the values of $g$ as in the statement, since 1-nodal curves are known to exist in $|H|$ for a general primitively polarized $(S, H)$ of any genus $g+1 \geq 2[\mathrm{MM}$, Ch].

Now a few words about the method of proof. Letting $P \in S$ be the unique singular point of $\widetilde{C}$ we consider the blow-up $\sigma: X:=\mathrm{Bl}_{P} S \longrightarrow S$ at $P$ and we let $E \subset X$ be the exceptional curve. Then the normalization of $\widetilde{C}$ is the strict transform $C=\sigma^{*} \widetilde{C}-2 E \subset X$. The Wahl map $\Phi_{K}$ on $C$ can be decomposed as

$$
\Phi_{K}=H^{0}(\rho) \cdot \Phi_{K_{X}+C}
$$

where $\Phi_{K_{X}+C}$ is a gaussian map on $X$ and $H^{0}(\rho)$ is induced in cohomology by a restriction homomorphism:

$$
\rho: \Omega_{X}^{1}\left(2 K_{X}+2 C\right) \longrightarrow \omega_{C}^{3}
$$

on $X$. We study these two maps and prove their surjectivity separately. This method of proof is analogous to the one adopted in the work of several authors before, notably BM, CLM1, CLM2, DM, W2. The restriction on the genus depends on the proof: one would expect the result to hold for $g=10$ (as it does indeed, as already remarked) and for $g \geq 12$. In fact the surjectivity of $H^{0}(\rho)$ holds for $g=10$ or $g \geq 12$ (Lemma 3). On the other hand the proof of the surjectivity of $\Phi_{K_{X}+C}$, which consists in adapting an analogous proof given in [CLM2] for plane curves, leads to the restrictions on $g$ in Theorem 1, indeed this proof requires that we decompose a certain divisor on $X$ as the sum of three very ample ones and this decomposition forces the genus to increase.

Recent work by M. Kemeny [Ke implies that the curves $C$ considered here, i.e. normalizations of 1-nodal curves on a general primitive K3 surface, are generically Brill-Noether-Petri general and fill a locus in $\mathrm{M}_{g}$, the coarse moduli space of curves of genus $g$, whose closure has dimension $19+g$. Theorem 1 and W1 imply that this naturally defined locus is not contained in the closure of the so-called K3-locus (i.e. the locus of smooth curves that can be embedded in a K3 surface).

One can ask whether a result analogous to Theorem 1 can be proved for the normalization of curves on K3 surfaces having a more complicated singular point. We did not consider this case. Note though that, to our knowledge, such curves are known to exist only in the case of $A_{k}$-singularities or ordinary triple points (see GK, Ga]).

The paper is organized as follows. In 11 we introduce the gaussian maps and explain the strategy of proof of the surjectivity of the Wahl map of a curve lying in a regular surface. In 92 we prove the surjectivity of $H^{0}(\rho)$ and in 93 we prove the surjectivity of $\Phi_{K_{X}+C}$. We work over $\mathbb{C}$.

\section{Generalities on Gaussian maps}

In this section we recall a few definitions and basic facts concerning gaussian maps. Given line bundles $L, M$ on a nonsingular projective variety $Y$ we consider:

$$
\mathcal{R}(L, M)=\operatorname{ker}\left[H^{0}(Y, L) \otimes H^{0}(Y, M) \rightarrow H^{0}(Y, L \otimes M)\right]
$$


Then we have a canonical map:

$$
\Phi_{L, M}: \mathcal{R}(L, M) \longrightarrow H^{0}\left(Y, \Omega_{Y}^{1} \otimes L \otimes M\right)
$$

called the gaussian map, or Wahl map, of $L, M$, which is defined as follows. Let $\Delta \subset Y \times Y$ be the diagonal and $p_{1}, p_{2}: Y \times Y \rightarrow Y$ the projections. Then

$$
\mathcal{R}(L, M)=H^{0}\left(Y \times Y, p_{1}^{*} L \otimes p_{2}^{*} M \otimes \mathcal{I}_{\Delta}\right)
$$

Since $\mathcal{I}_{\Delta} \otimes \mathcal{O}_{\Delta}=\Omega_{Y}^{1}$, the restriction to $\Delta$ :

$$
p_{1}^{*} L \otimes p_{2}^{*} M \otimes \mathcal{I}_{\Delta} \longrightarrow p_{1}^{*} L \otimes p_{2}^{*} M \otimes \mathcal{I}_{\Delta} \otimes \mathcal{O}_{\Delta}
$$

induces $\Phi_{L, M}$ on global sections. The exact sequence:

$$
0 \longrightarrow p_{1}^{*} L \otimes p_{2}^{*} M \otimes \mathcal{I}_{\Delta}^{2} \longrightarrow p_{1}^{*} L \otimes p_{2}^{*} M \otimes \mathcal{I}_{\Delta} \longrightarrow p_{1}^{*} L \otimes p_{2}^{*} M \otimes \mathcal{I}_{\Delta} \otimes \mathcal{O}_{\Delta} \longrightarrow 0
$$

shows that the vanishing:

$$
H^{1}\left(Y \times Y, p_{1}^{*} L \otimes p_{2}^{*} M \otimes \mathcal{I}_{\Delta}^{2}\right)=0
$$

is a sufficient condition for the surjectivity of $\Phi_{L, M}$.

In case $L=M$ we have $\mathcal{R}(L, L)=I_{2}(Y) \oplus \bigwedge^{2} H^{0}(Y, L)$, where

$$
I_{2}(Y)=\operatorname{ker}\left[S^{2} H^{0}(Y, L) \rightarrow H^{0}\left(Y, L^{2}\right)\right]
$$

and $\Phi_{L, L}$ is zero on $I_{2}(Y)$. Therefore $\Phi_{L, L}$ is equivalent to its restriction to $\bigwedge^{2} H^{0}(Y, L)$, which is denoted by

$$
\Phi_{L}: \bigwedge^{2} H^{0}(Y, L) \longrightarrow H^{0}\left(Y, \Omega_{Y}^{1} \otimes L^{2}\right)
$$

In particular, for a non-hyperelliptic curve $C$ we are interested in $\Phi_{K, K}$ or rather in

$$
\Phi_{K}: \bigwedge^{2} H^{0}\left(C, \omega_{C}\right) \longrightarrow H^{0}\left(C, \omega_{C}^{3}\right)
$$

where $\mathcal{O}_{C}(K)=\omega_{C}$ is the canonical invertible sheaf.

Suppose that $C \subset X$ where $X$ is a nonsingular regular surface. Then the exact sequence:

$$
0 \rightarrow \mathcal{O}_{X}\left(K_{X}\right) \longrightarrow \mathcal{O}_{X}\left(K_{X}+C\right) \longrightarrow \omega_{C} \rightarrow 0
$$

shows that $H^{0}\left(X, K_{X}+C\right) \rightarrow H^{0}\left(C, \omega_{C}\right)$ is surjective. Moreover it is easy to show that $\Phi_{K}$ fits in the commutative diagram:

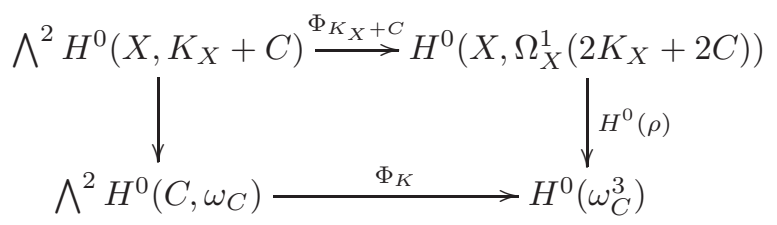

where

$$
\rho: \Omega_{X}^{1}\left(2 K_{X}+2 C\right) \rightarrow \omega_{C}^{3}
$$

is the restriction map. Since the left vertical map is surjective we have:

Lemma 2. In the above situation

$$
\operatorname{Im}\left(\Phi_{K}\right) \subset \operatorname{Im}\left(H^{0}(\rho)\right)
$$

and equality holds if $\Phi_{K_{X}+C}$ is surjective. In particular, if both $\Phi_{K_{X}+C}$ and $H^{0}(\rho)$ are surjective, so is $\Phi_{K}$. 


\section{The SurJeCtivity of $H^{0}(\rho)$}

As in the Introduction, we let $(S, H)$ be a $\mathrm{K} 3$ surface with a polarization of genus $g+1 \geq 3$ and let $\widetilde{C} \in|H|$ be a curve with one node, i.e. an ordinary double point, at $P \in S$ and no other singularities. Consider the blow-up $\sigma: X:=\mathrm{Bl}_{P} S \longrightarrow S$ of $S$ at $P$, let $E \subset X$ be the exceptional curve and $C=\sigma^{*} \widetilde{C}-2 E \subset X$ the strict transform of $\widetilde{C}$. We have an exact sequence on $X$ :

$$
0 \longrightarrow \Omega_{X}^{1}(\log C)(-C) \longrightarrow \Omega_{X}^{1} \longrightarrow \omega_{C} \longrightarrow 0
$$

where $\Omega_{X}^{1}(\log C)$ is the sheaf of 1 -forms with logarithmic poles along $C$ [EV]. Tensoring with $\mathcal{O}_{X}\left(2 K_{X}+2 C\right)$ we obtain:

$$
0 \longrightarrow \Omega_{X}^{1}(\log C)\left(2 K_{X}+C\right) \longrightarrow \Omega_{X}^{1}\left(2 K_{X}+2 C\right) \stackrel{\rho}{\longrightarrow} \omega_{C}^{3} \longrightarrow 0
$$

Lemma 3. Suppose that $(S, H)$ is a general primitively polarized K3 surface of genus $g+1$, with $g=10$ or $g \geq 12$. Then, with the same notations as above, we have:

$$
h^{1}\left(X, \Omega_{X}^{1}(\log C)\left(2 K_{X}+C\right)\right)=0
$$

In particular $H^{0}(\rho)$ is surjective.

Proof. The last assertion follows from the exact sequence (11). Since $K_{X}=E$ we have $\mathcal{O}_{X}\left(2 K_{X}+C\right)=\sigma^{*} H$. Consider the relative cotangent sequence of $\sigma$ :

$$
0 \longrightarrow \sigma^{*} \Omega_{S}^{1} \longrightarrow \Omega_{X}^{1} \stackrel{\eta}{\longrightarrow} \omega_{E} \longrightarrow 0
$$

and tensor it by $\sigma^{*} H$ :

$$
0 \longrightarrow \sigma^{*} \Omega_{S}^{1}(H) \longrightarrow \Omega_{X}^{1}\left(\sigma^{*} H\right) \stackrel{\eta_{H}}{\longrightarrow} \omega_{E} \longrightarrow 0
$$

We have:

$$
H^{2}\left(X, \sigma^{*} \Omega_{S}^{1}(H)\right)=H^{2}\left(S, \Omega_{S}^{1}(H)\right)=H^{0}\left(S, T_{S}(-H)\right)^{\vee}=0
$$

From the assumption about the genus and from the generality of $(S, H)$ it follows that we also have:

$$
H^{1}\left(X, \sigma^{*} \Omega_{S}^{1}(H)\right)=H^{1}\left(S, \Omega_{S}^{1}(H)\right)=0
$$

(see $[\mathrm{B}, 5.2)$. Therefore $\eta_{H}$ induces an isomorphism:

$$
H^{1}\left(\eta_{H}\right): H^{1}\left(X, \Omega_{X}^{1}\left(\sigma^{*} H\right)\right) \cong H^{1}\left(E, \omega_{E}\right) \cong \mathbb{C}
$$

Then in order to prove the lemma it suffices to show that in the exact sequence:

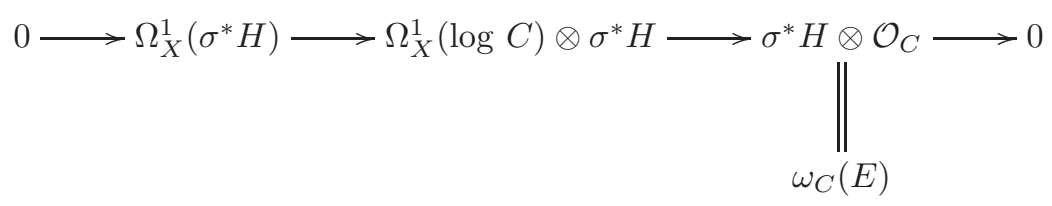

the coboundary map:

$$
\partial_{H}: H^{0}\left(C, \omega_{C}(E)\right) \longrightarrow H^{1}\left(X, \Omega_{X}^{1}\left(\sigma^{*} H\right)\right)
$$


is non-zero. The above sequence is part of the following exact and commutative diagram:

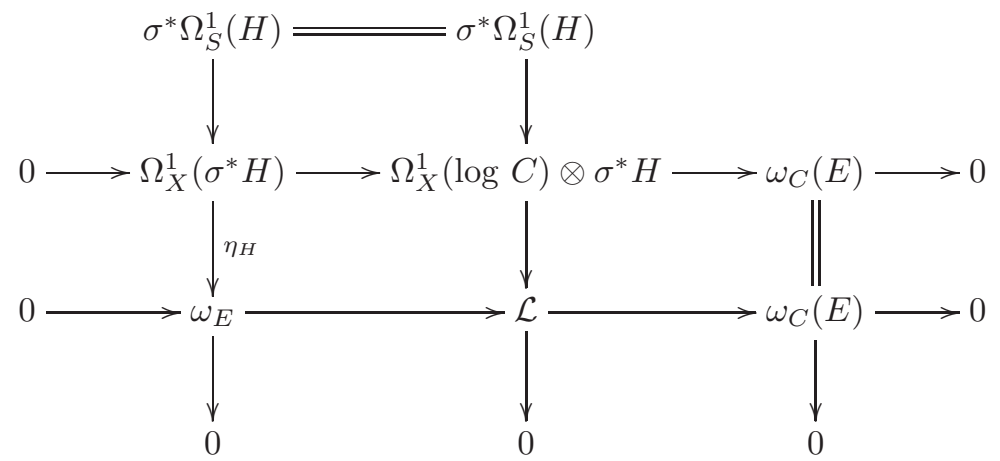

where $\mathcal{L}$ is an invertible sheaf on $C+E$. Since $H^{1}\left(\eta_{H}\right)$ is an isomorphism it suffices to show that the coboundary map $H^{0}\left(\omega_{C}(E)\right) \rightarrow H^{1}\left(\omega_{E}\right)$ of the last row is nonzero or, equivalently, that $H^{1}(\mathcal{L})=0$. Observe that $\mathcal{L}=\mathcal{M} \otimes \sigma^{*} H$, where $\mathcal{M}$ is defined by the following diagram:

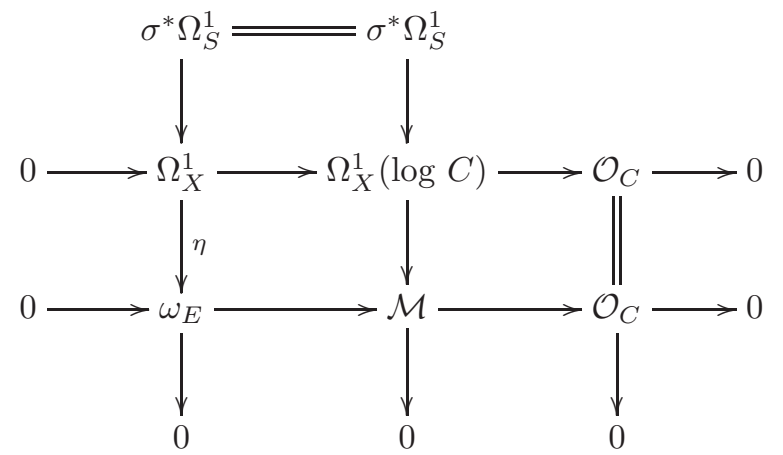

It suffices to show that $\mathcal{M} \neq \mathcal{O}_{C+E}$ because this will imply that $\mathcal{L} \neq \omega_{C+E}$, and in turn that $H^{1}(\mathcal{L})=0$. The coboundary map of the middle row

$$
\partial: H^{0}\left(C, \mathcal{O}_{C}\right) \longrightarrow H^{1}\left(X, \Omega_{X}^{1}\right)
$$

associates to $1 \in H^{0}\left(C, \mathcal{O}_{C}\right)$ the Atiyah-Chern class of $\mathcal{O}_{X}(C)$ and $H^{1}(\eta)(\partial(1))$ is its restriction to $E$. Moreover

$$
\operatorname{ker}\left(H^{1}(\eta)\right) \cong H^{1}\left(X, \sigma^{*} \Omega_{S}^{1}\right)
$$

is generated by the Atiyah-Chern classes of the total trasforms under $\sigma$ of curves in $S$, which are trivial when restricted to $E$. Since $E \cdot C=2$ we see that $\partial(1) \notin$ $\operatorname{ker}\left(H^{1}(\eta)\right)$. It follows that the coboundary of the last row:

$$
H^{0}\left(C, \mathcal{O}_{C}\right) \longrightarrow H^{1}\left(E, \omega_{E}\right)
$$

is non-zero. Hence $\mathcal{M} \neq \mathcal{O}_{C+E}$.

\section{The gaussian MAP ON $X$}

We keep the notations of 92 We will prove the following: 
Proposition 4. Suppose that $(S, H)$ is a general primitively polarized K3 surface of genus $g+1$, with $g=40,42$ or $\geq 44$; let $\widetilde{C} \in|H|$ be a 1-nodal curve and $C$ its normalization. Let $X=\operatorname{Bl}_{P}(S)$ where $P \in \widetilde{C}$ is the node. Then the gaussian map

$$
\Phi_{K_{X}+C}: \bigwedge^{2} H^{0}\left(X, \mathcal{O}_{X}\left(K_{X}+C\right)\right) \longrightarrow H^{0}\left(X, \Omega_{X}^{1}\left(2 K_{X}+2 C\right)\right)
$$

is surjective.

We will need the following result and its corollary:

Proposition 5. a) For every $d \geq 10$ there is a K3 surface $S$ containing two very ample nonsingular curves $A, B$ such that $\operatorname{Pic}(S)=\mathbb{Z}[A] \oplus \mathbb{Z}[B]$, with intersection matrix:

$$
\left(\begin{array}{cc}
A^{2} & A \cdot B \\
B \cdot A & B^{2}
\end{array}\right)=\left(\begin{array}{ll}
8 & d \\
d & 8
\end{array}\right)
$$

and $A$ and $B$ non-trigonal.

b) For every $d \geq 12$ there is a K3 surface $S$ containing two very ample nonsingular curves $A, B$ such that $\operatorname{Pic}(S)=\mathbb{Z}[A] \oplus \mathbb{Z}[B]$, with intersection matrix:

$$
\left(\begin{array}{cc}
A^{2} & A \cdot B \\
B \cdot A & B^{2}
\end{array}\right)=\left(\begin{array}{cc}
10 & d \\
d & 8
\end{array}\right)
$$

and $A$ and $B$ non-trigonal.

In both cases (a) and (b) the surface $S$ does not contain rational nonsingular curves $R$ such that $A \cdot R=1$ or $B \cdot R=1$.

Proof. The Proposition is a special case of $\underline{\mathrm{Kn}}$, Theorem 4.6. We obtain the Proposition by taking (with the notations used there) $(n, g-1)=(4,4)$ and $(n, g-$ $1)=(5,4)$ respectively. The restriction on $d$ is forced by the requirement that the hypotheses of the theorem apply symmetrically w.r. to $A$ an $B$ so that both are very ample. The non-trigonality follows from the fact that $S$ is embedded by both $|A|$ and $|B|$ so to be an intersection of quadrics. The last assertion is proved as done in loc. cit. for $(-2)$ curves, by comparing discriminants.

Corollary 6. If $S$ is as in Prop. 5(a) then $H=A+2 B$ defines a primitive very ample divisor class of genus $g+1=21+2 d$ for every $d \geq 10$. If $S$ is as in Prop. 5(b) then $H=A+2 B$ defines a primitive very ample divisor class of genus $g+1=22+2 d$ for every $d \geq 12$.

Proof. Clearly $H$ is very ample and it is primitive because the generator $A$ of $\operatorname{Pic}(S)$ appears with coefficient 1 . The genus in either case is readily computed using the intersection matrix.

Proof. of Proposition 4 By semicontinuity it suffices to prove the Proposition for just one primitively polarized K3 surface for each value of $g$ as in the statement. We take $(S, H)$ as in Corollary 6 , distinguishing cases (a) and (b) according to the parity of $g$. Letting $\widetilde{C}, C, P$ and $X$ as in the statement, consider the product $X \times X$ and the blow-up $\pi: Y=\mathrm{Bl}_{\Delta}(X \times X) \rightarrow X \times X$ along the diagonal $\Delta$. Let $\Lambda \subset Y$ be the exceptional divisor. For any coherent sheaf $\mathcal{F}$ on $X$ we define $\mathcal{F}_{i}=\left(p_{i} \cdot \pi\right)^{*} \mathcal{F}$, $i=1,2$. It suffices to prove that

$$
H^{1}\left(X \times X, p_{1}^{*} \mathcal{O}_{X}\left(K_{X}+C\right) \otimes p_{2}^{*} \mathcal{O}_{X}\left(K_{X}+C\right) \otimes \mathcal{I}_{\Delta}^{2}\right)=0
$$


which is equivalent to:

$$
H^{1}\left(Y,\left(K_{X}+C\right)_{1}+\left(K_{X}+C\right)_{2}-2 \Lambda\right)=0
$$

Note that we have:

$$
\left(K_{X}+C\right)_{1}+\left(K_{X}+C\right)_{2}-2 \Lambda=K_{Y}+C_{1}+C_{2}-3 \Lambda
$$

and therefore we want to prove that:

$$
H^{1}(Y, \mathcal{L})=0
$$

where we set $\mathcal{L}=\mathcal{O}_{Y}\left(K_{Y}+C_{1}+C_{2}-3 \Lambda\right)$. The proof is an adaptation of the proofs of Lemmas (3.1) and (3.10) of [CLM2]. One uses the following:

Lemma 7. Assume that $D$ is a very ample divisor on $X$. Then $D_{1}+D_{2}-\Lambda$ is big and nef on $Y$.

Proof. See [BEL, Claim 3.3.

Let $M:=\sigma^{*} H(-3 E)$ on $X$. We have:

$$
M=\sigma^{*} A(-E)+2\left(\sigma^{*} B(-E)\right)
$$

Since $A$ and $B$ are non-trigonal $S$ has no trisecant lines through $P$ and does not contain a line through $P$ whether it is embedded by $|A|$ or by $|B|$ (Proposition 55). Therefore every curvilinear subscheme of $S$ of length 3 containing $P$ imposes independent conditions to both $|A|$ and $|B|$. Then it follows from $[\mathrm{Co}$, Prop. 1.3.4, that both $\sigma^{*} A(-E)$ and $\sigma^{*} B(-E)$ are very ample on $X$. Therefore by the Lemma we have that both $\sigma^{*} A(-E)_{1}+\sigma^{*} A(-E)_{2}-\Lambda$ and $\sigma^{*} B(-E)_{1}+\sigma^{*} B(-E)_{2}-\Lambda$ are big and nef. It follows that the divisor $M_{1}+M_{2}-3 \Lambda$ is big and nef, being the sum of three big and nef divisors.

Since $C \sim M+E$ we have $C_{1}+C_{2} \sim M_{1}+M_{2}+E_{1}+E_{2}$ and therefore we have an exact sequence on $Y$ :

$$
0 \rightarrow \mathcal{O}_{Y}\left(K_{Y}+M_{1}+M_{2}-3 \Lambda\right) \rightarrow \mathrm{L} \rightarrow \mathcal{O}_{E_{1}+E_{2}}\left(\mathrm{~L}_{s}\right) \rightarrow 0
$$

By Kawamata-Vieweg we have $H^{1}\left(Y, K_{Y}+M_{1}+M_{2}-3 \Lambda\right)=0$ : therefore in order to prove (3) it suffices to show that

$$
H^{1}\left(E_{1}+E_{2}, \mathcal{O}_{E_{1}+E_{2}}(\mathrm{~L})\right)=0
$$

Letting $W:=E_{1} \cap E_{2}$ we have an exact sequence:

$$
0 \rightarrow \mathcal{O}_{E_{1}}(\mathcal{L}-W) \rightarrow \mathcal{O}_{E_{1}+E_{2}}(\mathcal{L}) \rightarrow \mathcal{O}_{E_{2}}(\mathcal{L}) \rightarrow 0
$$

and, by symmetry, it suffices to prove that:

$$
H^{1}\left(E_{1}, \mathcal{O}_{E_{1}}(\mathcal{L}-W)\right)=0
$$

and

$$
H^{1}\left(E_{1}, \mathcal{O}_{E_{1}}(\mathcal{L})\right)=0
$$

We can then consider the exact sequence on $E_{1}$ :

$$
0 \rightarrow \mathcal{O}_{E_{1}}(\mathcal{L}-W) \rightarrow \mathcal{O}_{E_{1}}(\mathcal{L}) \rightarrow \mathcal{O}_{W}(\mathcal{L}) \rightarrow 0
$$

and finally we are reduced to prove (5) and

$$
H^{1}\left(W, \mathcal{O}_{W}(\mathcal{L})\right)=0
$$

Let $U \cong E \times E$ be the proper transform of $E \times E$ in $Y$. Then in $E_{1}$ we have $W=U+\Lambda_{\mid E_{1}}$. As in [CLM2], proof of Lemma (3.1), one shows that $\Lambda_{\mid E_{1}} \cong \mathbb{P} \mathcal{E}$, where $\mathcal{E}=\mathcal{O}_{\mathbb{P}^{1}}(1) \oplus \mathcal{O}_{\mathbb{P}^{1}}(-2)$, and $\mathcal{L}_{\mid \mathbb{P} \mathcal{E}}=\mathcal{O}_{\mathbb{P} \mathcal{E}}\left(2 C_{0}+2 f\right)$ where $C_{0} \in\left|\mathcal{O}_{\mathbb{P} \mathcal{E}}(1)\right|$ and 
$f$ is a fibre of $\mathbb{P} \mathcal{E} \rightarrow \mathbb{P}^{1}$. Now the proof proceeds as in CLM2, Lemma (3.1), after having proved that $\mathcal{O}_{E_{1}}(\mathcal{L}-W)=\mathcal{O}_{E_{1}}\left(K_{Y}+M_{1}+M_{2}-3 \Lambda\right)$ is big and nef. This last fact is obtained exactly as in the proof of Lemma (3.10) of [CLM2, using the fact that both $\sigma^{*} A(-E)$ and $\sigma^{*} B(-E)$ are very ample on $X$.

Proof. of Theorem 1, Recalling Lemma 2, the theorem follows immediately from Lemma 3 and from Proposition 4

\section{REFERENCES}

[BF] Ballico E., Fontanari C.: Gaussian maps, the Zak map and projective extensions of singular varieties. Result. Math. 44 (2003), 29-34.

[BFT] Ballico E., Fontanari C., Tasin L.: Singular curves on K3 surfaces. Sarajevo J. of Math. 6 (2010), 165-168.

[B] Beauville A.: Fano threefolds and K3 surfaces. Proceedings of the Fano Conference Torino 2002. Edited by A. Collino, A. Conte, M. Marchisio (2004).

[BM] Beauville A., Merindol J.Y.: Sections hyperplanes des surfaces K3. Duke Math. J. 55 (1987), 873-878.

[BEL] Bertram A., Ein L., Lazarsfeld R.: Vanishing theorems, a theorem of Severi, and the equations defining projective varieties. J. AMS 4 (1991), 587-602.

[Ch] Chen X.: Nodal curves on K3 surfaces. arXiv:1611.07423.

[CHM] Ciliberto C., Harris J., Miranda R.: On the surjectivity of the Wahl map. Duke Math. J. 57 (1988), 829-858.

[CK] Ciliberto C., Knutsen A.L.: On k-gonal loci in Severi varieties on general K3 surfaces and rational curves on hyperkähler manifolds. J. Math. Pures Appl. (9) 101 (2014), no. 4, 473-494.

[CLM1] Ciliberto C., Lopez A.F., Miranda R.: On the corank of gaussian maps for general embedded K3 surfaces. In Israel Mathematical Conference Proceedings. Papers in honor of Hirzebruch's 65th birthday, vol. 9, AMS Publications (1996), 141-157.

[CLM2] Ciliberto C., Lopez A.F., Miranda R.: On the Wahl map of plane nodal curves. In Complex Analysis and Algebraic Geometry, a volume in Memory of Michael Schneider. Thomas Peternell and Frank-Olaf Schreyer, editors. Walter de Gruyter, Berlin/New York 2000. p. 155-163.

[Co] Coppens M.: Very ample linear systems on blowings-up at general points of smooth projective varieties. Pacific J. Math. 202 (2002), 313-327.

[DM] Duflot J., Miranda R.: The gaussian map for rational ruled surfaces. Trans. AMS 330 (1992), 447-459.

[EV] H. Esnault, E. Viehweg: Lectures on Vanishing Theorems, DMV Seminar, vol. 20, Birkhauser Verlag (1992).

[FKP] Flamini F., Knutsen A.L., Pacienza G.: Singular curves on a K3 surface and linear series on their normalizations. International J. of Math. 18 (2007), 671-693.

[FKPS] Flamini F., Knutsen A.L., Pacienza G., Sernesi E.: Nodal curves with general moduli on K3 surfaces. Comm. in Algebra 36 (2008), 3955-3971.

[Ga] Galati, C.: On the existence of curves with a triple point on a K3 surface. Atti Accad. Naz. Lincei Rend. Lincei Mat. Appl. 23 (2012), no. 3, 295 ?317.

[GK] Galati, C., Knutsen, A.L.: On the existence of curves with $A_{k}$-singularities on K3 surfaces. Math. Res. Lett. 21 (2014), no. 5, 1069-1109.

[Go] Gómez, T. L.: Brill-Noether theory on singular curves and torsion-free sheaves on surfaces. Comm. Anal. Geom. 9 (2001), no. 4, 725-756.

[Hal1] Halic M.: Modular properties of nodal curves on K3 surfaces. Math. Z. 270 (2012), 871-887.

[Hal2] Halic M.: Erratum to: Modular properties of nodal curves on K3 surfaces. Math. Z. 280 (2015), 1203-1211.

[Har] Hartshorne R.: Algebraic Geometry. Graduate Texts in Mathematics 52. Springer Verlag, New York-Heidelberg-Berlin 1977.

[Ke] Kemeny M.: The moduli of singular curves on K3 surfaces. J. de Math. Pures et appliquées 104 (2015), 882-920. 
[Kn] Knutsen A.L.: Smooth curves on projective K3 surfaces. Mathematica Scandinavica 90 (2002) 215-231.

[L] Lazarsfeld R.: Brill-Noether-Petri without degenerations. J. Diff. Geom. 23 (1986) 299-307.

[MM] Mori S., Mukai S.: The uniruledness of the moduli space of curves of genus 11. Algebraic Geometry. Proceedings Tokyo/Kyoto, 334-353. Springer LNM 1016 (1983).

[W1] Wahl J.: The jacobian algebra of a graded Gorenstein singularity. Duke Math. J. 55 (1987), 843-872.

[W2] Wahl J.: Gaussian maps on algebraic curves. J. Diff. Geometry 32 (1990), 77-98.

E-mail address: sernesi@mat.uniroma3.it

Dipartimento di Matematica e Fisica, Università Roma Tre, L.go S.L. Murialdo 1, 00146 ROMA.

E-mail address: sernesi@gmail.com 